\title{
Erratum to: PRIMA 2016: Principles and Practice of Multi-Agent Systems
}

\author{
Matteo Baldoni ${ }^{1(凶)}$, Amit K. Chopra ${ }^{2}$, Tran Cao Son ${ }^{3}$, \\ Katsutoshi Hirayama ${ }^{4}$, and Paolo Torroni ${ }^{5}$ \\ ${ }^{1}$ Dipartimento di Informatica, Università degli Studi di Torino, Turin, Italy \\ baldoni@di.unito.it \\ 2 Computing and Communications, Lancaster University, Lancaster, UK \\ 3 Department of Computer Science, New Mexico State University, \\ Las Cruces, NM, USA \\ ${ }^{4}$ Graduate School of Maritime Sciences, Kobe University, Kobe, Japan \\ 5 Dept. di Informatica: Sci. e Ingegneria, Universitá di Bologna, Bologna, Italy
}

\section{Erratum to: \\ M. Baldoni et al. (Eds.) \\ PRIMA 2016: Principles and Practice of Multi-Agent Systems DOI: $10.1007 / 978-3-319-44832-9$}

The original version of the cover and title page was revised: The conference title is updated. Title of the conference is updated from PRIMA 2016: Princiles and Practice of Multi-Agent Systems to PRIMA 2016: Principles and Practice of Multi-Agent Systems.

The updated original online version for this book can be found at 10.1007/978-3-319-44832-9 